\title{
El lado oscuro de la amiodarona
}

\author{
María Amparo Vicente-Altabás', Lucía Martínez-Barredo', Luis Ignacio Fumanal-Idocin', Anyuli Gracia-Gutiérrez² \\ ${ }^{1}$ Servicio de Medicina Interna. Hospital Universitario Miguel Servet. Zaragoza. España \\ ${ }^{2}$ Servicio de Medicina Interna. Hospital General de la Defensa. Zaragoza. España
}

Recibido: 18/11/2019

Aceptado: 22/04/2020

En línea: 31/08/2020

Citar como: Vicente-Altabás MA, Martínez-Barredo L, Fumanal-Idocin LI, Gracia-Gutiérrez A. El lado oscuro de la amiodarona. Rev Esp Casos Clin Med Intern (RECCMI). 2020 (Ago); 5(2): 59-61. doi: 10.32818/reccmi.a5n2a2.

Cite this as: Vicente-Altabás MA, Martínez-Barredo L, Fumanal-Idocin LI, Gracia-Gutiérrez A. The dark side of amiodarone. Rev Esp Casos Clin Med Intern (RECCMI). 2020 (Ago); 5(2): 59-61. doi: 10.32818/reccmi.a5n2a2.

Autor para correspondencia: María Amparo Vicente-Altabás.a.vicentealtabas@gmail.com

\section{Palabras clave \\ $\triangleright$ Amiodarona \\ $\triangleright$ Efecto adverso medicamentoso \\ $\triangleright$ Enfermedad pulmonar}

Keywords

$\triangleright$ Amiodarone
$\triangleright$ Adverse drug reaction
$\triangleright$ Lung disease

$\triangleright$ Adverse drug reaction

\section{Resumen}

La toxicidad pulmonar por amiodarona es una entidad poco frecuente, pero grave. Su diagnóstico es difícil debido a que la clínica es inespecífica y a la necesidad de descartar otros procesos que simulan esta patología. Es importante un alto grado de sospecha, para obtener un diagnóstico temprano y un tratamiento precoz, evitando su progresión a fibrosis pulmonar. Presentamos un caso que ilustra la dificultad diagnóstica y la buena evolución clínica y radiológica tras la retirada de amiodarona.

Abstract
Amiodarone pulmonary toxicity is a rare but serious entity. Its diagnosis is difficult because the clinic is nonspecific
and the need to rule out other processes that simulate this pathology. A high degree of suspicion is important, to
obtain an early diagnosis and early treatment, avoiding its progression to pulmonary fibrosis. We present a case
that illustrates the diagnostic difficulty and the good clinical and radiological evolution after the withdrawal of
amiodarone.

\section{Puntos destacados}

$\triangleright$ El tratamiento con amiodarona es frecuente en nuestro medio y es importante conocer sus efectos adversos, entre ellos a nivel pulmonar.

$\triangleright$ Un alto grado de sospecha de los mismos permite un diagnóstico precoz y un tratamiento temprano, evitando complicaciones posteriores.

\section{Introducción}

La toxicidad pulmonar por amiodarona es un efecto secundario poco frecuente, pero grave, de este fármaco. Se correlaciona estrechamente con la dosis total acumulada más que con los niveles en suero. Son múltiples las formas de presentación clínica, como la afectación intersticial pulmonar (la más frecuente), la neumonía organizada, el síndrome de distrés respiratorio del adulto, la hemorragia alveolar difusa e, incluso, los nódulos pulmonares y el derrame pleural. En ocasiones, su diagnóstico es difícil y se basa en la clínica con la exclusión de otras posibilidades diagnósticas, tales como procesos tumorales, infecciosos, autoinmunes o cardiológicos. Es considerable la mejoría clínica y radiológica que experimentan los pacientes tras la suspensión del fármaco. Su tratamiento consiste en la retirada de la amiodarona y, en ocasiones, la aso- ciación de corticoterapia oral. La evolución suele ser favorable, aunque hay algún caso que progresa hacia la fibrosis pulmonar.

El objetivo de este caso clínico es mostrar la importancia de la sospecha clínica de esta entidad, puesto que diariamente vemos a un porcentaje no despreciable de pacientes que están en tratamiento con este fármaco.

\section{Caso clínico}

Mujer de 67 años de edad, no fumadora, ama de casa. Como antecedentes personales relevantes destacaban hipertensión arterial que controlaba con medidas higiénico-dietéticas; hipotiroidismo en tratamiento sustitutivo, como consecuencia de la enfermedad de Graves-Basedow y su tratamiento con iodo radiactivo; fibrilación auricular paroxística sin anticoagular, en tratamiento con amiodarona 200 mg/día desde hacía 8 años; aplastamiento vertebral L1 y extirpación de tumoración glómica en talón derecho.

Acudía a Urgencias por presentar aumento progresivo de disnea hasta hacerse de mínimos esfuerzos, de 10 días de evolución. No ortopnea ni edemas en extremidades inferiores. Se acompañaba de escasa tos sin expectoración. 
No tenía dolor torácico ni sensación de palpitaciones. Afebril. Negaba síndrome constitucional. Sin contacto con animales. A la auscultación pulmonar destacaban crepitantes bibasales.

En las pruebas complementarias iniciales, se observó un ritmo sinusal en el electrocardiograma; proBNP negativo, proteína $C$ reactiva $(P C R)$ 1,95 mg/dl $(0,0-0,5)$ y hemograma normal en la analítica sanguínea; y patrón intersticialnodular bibasal informado como posible linfangitis carcinomatosa en la radiografía de tórax (Figura 1).

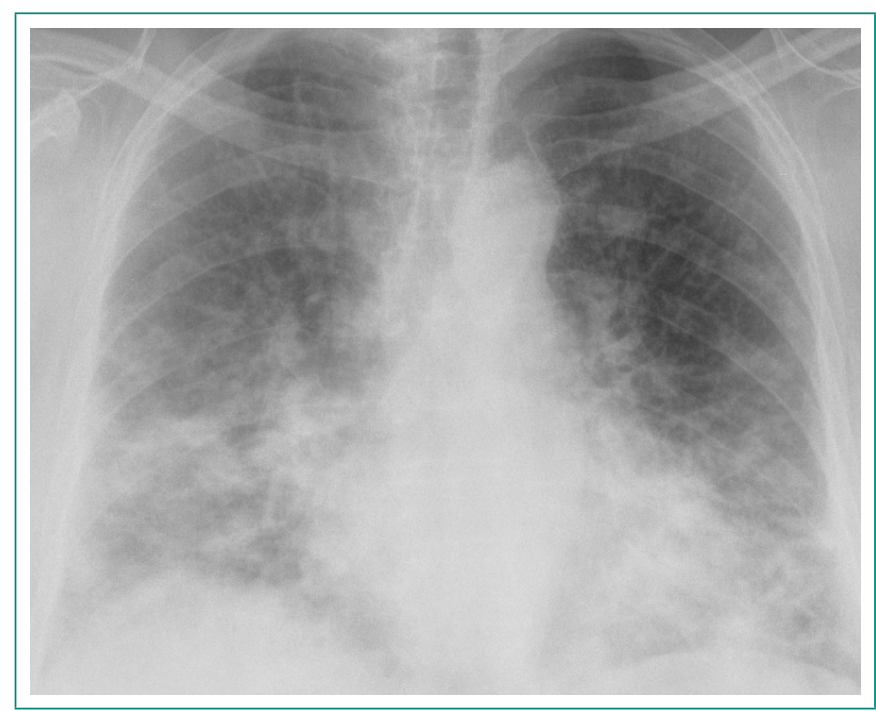

Figura 1. Radiografía de tórax al ingreso

Se decidió su ingreso en el Servicio de Medicina Interna para tratamiento y completar estudio. El diagnóstico diferencial inicial que se planteó fue afectación pulmonar de causa tumoral, infecciosa, farmacológica por amiodarona o cardiológica. Se solicitó autoinmunidad, serología VIH, tres cultivos de esputo seriados, resultando ser todo ello negativo. El ecocardiograma fue normal. Se amplió el estudio con una tomografía computarizada (TC) torácica y abdominal, siendo informada como múltiples infiltrados pulmonares bilaterales pseudonodulares mal definidos en campos medios e inferiores que asociaba engrosamiento de septos, junto con un aumento difuso de la densidad hepática (Figura 2).

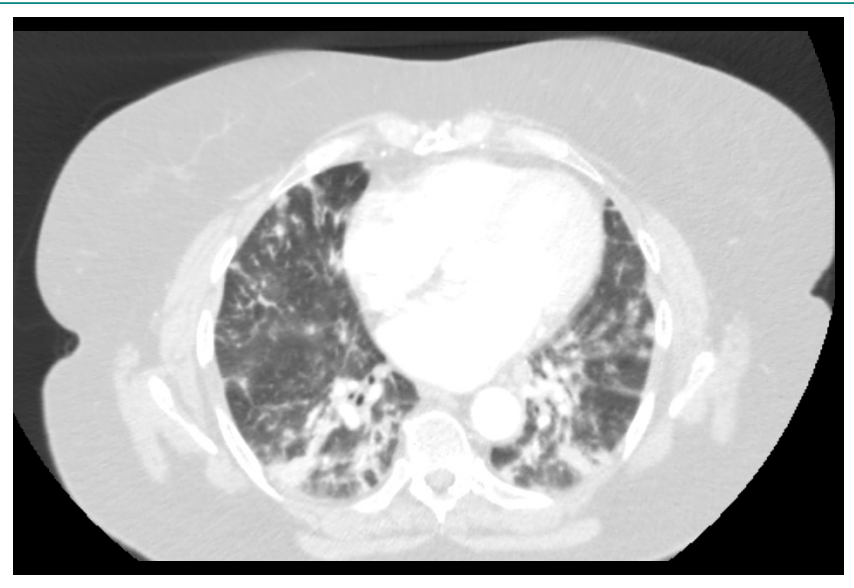

Figura 2. TC torácica en paciente con toxicidad pulmonar por amiodarona

Se realizó una fibrobroncoscopia, sin objetivarse lesiones endobronquiales, y la citología del lavado broncoalveolar fue negativa para malignidad, objeti- vándose predominio de macrófagos alveolares. Ante la alta sospecha clínica de afectación pulmonar por amiodarona, se decidió suspender y se instauró tratamiento con corticoides. La paciente presentó entonces una mejoría clínica y radiológica importantes (Figura 3), y se le dio el alta con flecainida y edoxabán, y con seguimiento posterior.

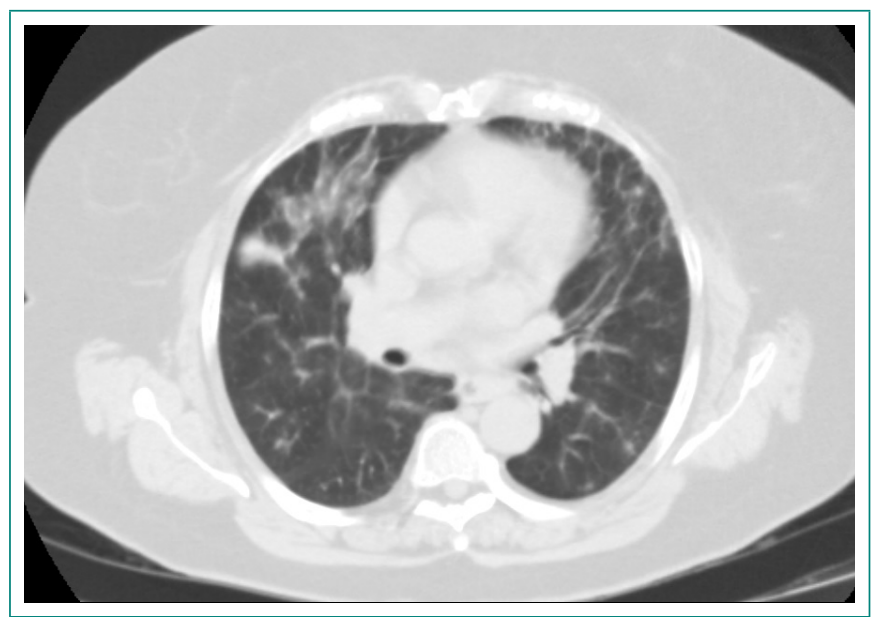

Figura 3. TC torácica tras 10 días sin amiodarona

Valorada 2 meses más tarde en Consultas, se constató la gran mejoría clínica de la paciente.

\section{Discusión}

La amiodarona es un fármaco antiarrítmico ampliamente utilizado en varios tipos de taquiarritmias, tanto supraventriculares como ventriculares. Son numerosos sus efectos adversos, siendo la afectación pulmonar uno de los más graves, con una incidencia del 1-5\% . Dentro de ella se incluyen la afectación intersticial pulmonar, la neumonía organizada, el síndrome de distrés respiratorio del adulto, la hemorragia alveolar difusa, los nódulos pulmonares e incluso el derrame pleural. La enfermedad pulmonar intersticial es la forma más común de presentación de toxicidad pulmonar por amiodarona. Los mecanismos patogénicos implicados son todavía desconocidos, pero desempeñan un papel importante la hipersensibilidad al fármaco y la citotoxicidad directa. Los factores de riesgo asociados a su aparición son una mayor dosis de fármaco acumulada, bien sea por un tiempo prolongado de tratamiento o por una dosis diaria administrada más elevada; mayor edad; coexistencia de una enfermedad pulmonar; procedimientos quirúrgicos y angiografía pulmonar ${ }^{1}$. En cuanto a la presentación clínica, suele ser inespecífica, con disnea progresiva, tos no productiva y, ocasionalmente, fiebre ${ }^{2}$.

Para el diagnóstico, es importante descartar otros procesos cardiológicos, infecciosos, autoinmunes o tumorales. La determinación de niveles de amiodarona en sangre no tiene utilidad predictiva ni diagnóstica. Se propuso la determinación del marcador KL-6, pero carece de especificidad ${ }^{3}$. En relación con la imagen radiológica, en la mayoría de los casos encontramos un patrón radiológico intersticial difuso bilateral de predominio periférico. Son más infrecuentes las imágenes radiológicas bilaterales alveolointersticiales confluentes difusas o el patrón alveolar tipo parcheado de distribución periférica, simulando una neumonía. La imagen de la TC muestra áreas de mayor densidad pulmonar, hepática y esplénica, debido al acúmulo de amiodarona en los macrófagos tisulares de estos órganos. Estos hallazgos son específicos del uso de amiodarona, pero no diagnósticos de toxicidad pulmonar ${ }^{4}$. En cuanto a las pruebas de función respiratoria, objetivamos un patrón restrictivo y una disminución en 
la capacidad de difusión, que no son específicos. La presencia de macrófagos vacuolados es frecuente en el lavado broncoalveolar y en la biopsia pulmonar (no necesaria en la mayoría de los casos), pero no es patognomónico, ya que también aparece en pacientes expuestos al fármaco que no desarrollarán toxicidad pulmonar 5 .

El tratamiento consiste en suspender la amiodarona y asociar corticoterapia vía oral (prednisona 40-60 mg/día con pauta descendente progresiva) en los pacientes más sintomáticos. La retirada del fármaco no siempre va seguida de una mejoría inmediata debido a la larga vida media de la amiodarona por su acúmulo en los tejidos grasos. Se recomienda no reiniciar el tratamiento con amiodarona una vez solucionado el cuadro, puesto que existe mayor riesgo de recurrencia y de progresión hacia fibrosis pulmonar. En general, su pronóstico es favorable una vez que se ha suspendido el fármaco. Sin embargo, su evolución en el tiempo puede conducir hacia la fibrosis pulmonar (hasta $5-7 \%$ de los casos) $)^{5}$

\section{Conclusión}

La toxicidad por amiodarona es una entidad poco frecuente pero grave. Su diagnóstico es difícil, debido a la poca especificidad de los síntomas y de los resultados de las pruebas complementarias, y, por tanto, se retrasa con fre- cuencia su supresión si no existe una fuerte sospecha clínica. Por ello, es importante su conocimiento para poder instaurar otro tratamiento precozmente y evitar su progresión.

\section{Bibliografía}

1. Papiris SA, Triantafillidou C, Kolilekas K, Markoulaki D, Manali ED. Amiodarone: review of pulmonary effects and toxicity. Drug Saf. 2010; 33(7): 539-558. doi: 10.2165/11532320-000000000-00000

2. Sánchez S, Zamora E, Hoyos N, Naranjo A, Al Nakeeb Z, Girón RM. Fibrosis pulmonar por amiodarona. Rev Patol Respir. 2006; 9(2): 77-80. Accesible en: https://www.revistadepatologiarespiratoria.org/descargas/pr_9-2_77-80. pdf (último acceso, 19-11-2019).

3. Bernal Morell E, Hernández Madrid A, Marín Marín I, Rodríguez Pena R, González Gordaliza M, Moro C. Nódulos pulmonares múltiples y amiodarona. KL-6 como nueva herramienta diagnóstica. Rev Esp Cardiol. 2005; 58(4): 447-449. doi: 10.1157/13073900.

4. Bahena López E, Bucio Reta E, Bahena López J. Toxicidad pulmonar por amiodarona. Rev Invest Med Sur Mex. 2015; 22(4): 214-217. Accesible en: https://www.medigraphic.com/pdfs/medsur/ms-2015/ms154k.pdf (último acceso, 19-11-2019)

5. Wolkove N, Baltzan M. Amiodarone pulmonary toxicity. Can Respir J. 2009; 16(2): 43-48. doi: 10.1155/2009/282540. 